\title{
Des fantômes dans la bibliothèque. L'Antiquité dans le Manuscrit trouvé à Saragosse
}

Danielle van Mal-Maeder

\section{(2) OpenEdition \\ 1 Journals}

Édition électronique

URL : http://journals.openedition.org/edl/452

DOI : $10.4000 /$ edl. 452

ISSN : 2296-5084

Éditeur

Université de Lausanne

\section{Édition imprimée}

Date de publication : 15 décembre 2012

Pagination : 27-42

ISBN : 978-2-940331-29-7

ISSN : 0014-2026

Référence électronique

Danielle van Mal-Maeder, «Des fantômes dans la bibliothèque. L'Antiquité dans le Manuscrit trouvé à Saragosse », Études de lettres [En ligne], 4 | 2012, mis en ligne le 15 décembre 2015, consulté le 18 décembre 2020. URL : http://journals.openedition.org/edl/452 ; DOI : https://doi.org/10.4000/edl.452 


\section{DES FANTÔMES DANS LA BIBLIOTHÈQUE. L'ANTIQUITÉ DANS LE MANUSCRIT TROUVÉ À SARAGOSSE*}

Le Manuscrit trouvé à Saragosse présente des correspondances intrigantes avec la littérature antique, notamment, du point de vue des motifs et des techniques narratives, avec les Métamorphoses d'Apulée. Néanmoins, les références précises sont rares. L'épisode de la bibliothèque dans le château d'Uzeda réveille toutefois quelques fantômes bien documentés, qui thématisent la question du livre-source (source d'information, de savoir, de divertissement) et proclament la texture livresque de l'œuvre.

This book had two authors, and they were both the same person.

Terry Pratchett, The Carpet People, Author's Note p. 7 (Corgi Books)

Le Manuscrit trouvé à Saragosse, œuvre du comte polonais Jean Potocki dont la vie constitue à elle seule un roman ${ }^{1}$, contient plusieurs références à l'Antiquité qui ont pour effet de thématiser la question du livre-source:

* Cette étude résulte d'une lectio magistralis donnée au «Seminario sulla continuità dell'anticho IV, 2010 : Io libro. Il libro come oggetto letterario nella cultura occidentale», Università Ca Foscari, Venezia. Elle a été publiée en italien dans la revue électronique CentoPagine IV, 2010, p. 1-10 (musacamena.units.it/iniziative/). Je remercie les éditeurs Gianfranco Agosti et Marco Fernandelli de m’avoir autorisée à en faire paraître ici la version française, quelque peu réduite dans sa première partie.

I. Sur la vie de Potocki, voir F. Rosset, D. Triaire, Jean Potocki, passim; voir aussi l'introduction à l'édition de Jean Potocki, Manuscrit trouvé à Saragosse par F. Rosset, D. Triaire, p. 7-56 (MTS-1804) et p. 3-54 (MTS-1810). 
source d'information, source de savoir, source de divertissement. On connaît bien l'histoire complexe du texte lui-même et de ses éditions successives, on sait aussi que le Manuscrit peut être regardé comme une véritable encyclopédie des formes et de la topique romanesques.

Pour la lectrice d'Apulée que je suis, les parallèles avec les Métamorphoses sont à première vue nombreux, à commencer par la technique narrative de base: un «je» y narre ses aventures selon la perspective limitée qui était la sienne dans le temps de l'histoire («je-narré»), sans anticipation, et de manière à placer le lecteur devant des alternatives interprétatives qui eussent fait les délices de John Winkler ${ }^{2}$. Le narrateur a beau connaître la fin de ses aventures (du moins dans la version de 1810), il ne fait pas usage de son savoir «d'après-coup ", ménageant ainsi la révélation finale; comme pour les Métamorphoses, le «lecteur second» ne portera jamais plus le même regard sur ce récit et cherchera les "signes" qui pourraient annoncer la fin de l'histoire ${ }^{3}$. Du point de vue thématique, Alphonse van Worden est, à l'image du Lucius d'Apulée, un jeune homme curieux, avide de nouvelles découvertes. Les péripéties de son voyage à travers la Sierra Morena qui sont narrées dans les premières journées du roman introduisent, dans un halo de surnaturel, ce qui constitue un thème majeur du Manuscrit trouvé à Saragosse - comme des Métamorphoses: le thème de la crédulité et de l'incrédulité, lié à celui des apparences et de la réalité, de la fiction et du mensonge:

Ce fait passait pour si certain qu'un théologien de Salamanque avait fait une dissertation dans laquelle il prouvait que les deux pendus étaient des espèces de vampires et que l'un n'était pas plus incroyable que l'autre, ce que les plus incrédules lui accordaient sans peine ${ }^{4}$.

2. J. Winkler, Auctor \& Actor.

3. Par «lecteur second», j'entends un lecteur ayant déjà lu le Manuscrit et qui le lit pour la seconde (ou la troisième) fois: son savoir est supérieur à celui qu'il possédait quand il découvrait le roman pour la première fois, supérieur aussi à celui des personnages de l'histoire. Son activité interprétative en est modifiée: voir D. van Mal-Maeder, Apuleius Madaurensis Metamorphoses, p. 9 sq. (à propos des Métamorphoses d'Apulée). Les notions de première lecture et de relecture sont introduites par R. Barthes, $S / Z$, p. 22 sq.

4. MTS-1804, p. 66, MTS-1810, p. 65. 
Les événements de la première journée du voyage d'Alphonse, avec la disparition de ses deux serviteurs, l'arrivée à la Venta Quemada, la cloche de minuit, l'irruption de la belle noire invitant le jeune homme à descendre dans les caves, la rencontre des deux cousines Emina et Zibeddé, tout cela n'est pas sans rappeler le premier livre des Métamorphoses où le dénommé Aristomène assiste à l'intrusion de deux sorcières dans sa chambre d'hôtel, dont l'une avait séduit son compagnon de route, Socrate ${ }^{5}$. Comme chez Apulée, l'épisode est relaté selon la perspective limitée du «je-narrant", de façon à accroître le suspense et à laisser planer le doute sur la véritable nature des deux femmes, qui se lancent dans une danse envoûtante:

Je les contemplai quelque temps avec une sorte de sang-froid; enfin leurs mouvements pressés par une cadence plus vive, le bruit étourdissant de la musique mauresque, mes esprits soulevés par une nourriture soudaine, en moi, hors de moi, tout se réunissait pour troubler ma raison. Je ne savais plus si j'étais avec des femmes ou bien avec $\mathrm{d}^{\prime}$ insidieux succubes ${ }^{6}$.

Passons sur les détails bien connus de la nuit passée par Alphonse en compagnie de ses cousines; au réveil, Alphonse fait ce constat effrayant: il est couché entre deux cadavres:

Les cadavres des deux frères de Zoto n'étaient point pendus, ils étaient couchés à mes côtés. J'avais apparemment passé la nuit avec eux. Je reposais sur des morceaux de cordes, des débris de roues, des restes de carcasses humaines et sur les affreux haillons que la pourriture en avait détachés ${ }^{7}$.

Ce réveil cauchemardesque peut être rapproché encore une fois de celui d'Aristomène qui, après avoir été témoin de l'assassinat de Socrate et de sa résurrection apparente - car tout cela paraît avoir été un rêve - est confronté le lendemain à la réalité de sa mort, qui survient de façon surnaturelle ${ }^{8}$.

5. Apulée, Métamorphoses 1.11-17.

6. MTS-1804 et 1810 , p. 72.

7. MTS-1804 et 1810 , p. 86.

8. Apulée, Métamorphoses 1.18-19. 
Ce qui se passe à la deuxième journée, en particulier avec l'irruption de Pascheco et de son histoire, enrichit encore la liste des rapprochements possibles. L'Antiquisant pensera à cet épisode, qui brode sur les motifs du récit-cadre, du dixième livre des Métamorphoses où, dans un récit enchâssé également, sévit une belle-mère de tragédie amoureuse de son beau-fils ${ }^{9}$. Quant à la scène de voyeurisme, elle rappelle le moment où Lucius assiste à la transformation de la sorcière Pamphilé en hibou, ou encore le passage du Satyricon de Pétrone dans lequel Quartilla invite Encolpe à épier l'union de Giton et de la jeune Pannychis ${ }^{10}$.

Le dialogue d'Alphonse avec l'ermite au cours duquel le jeune capitaine a l'occasion de raconter des scènes de son éducation, en particulier la lecture "pédagogique» de deux histoires de revenants, confirme l'observation déjà faite sur la prééminence du thème de la crédulité et de l'incrédulité, comme de celui des apparences et de la réalité, tous deux liés à la question de la source d'information: témoignage direct basé sur l'expérience personnelle (c'est le récit d'Alphonse et celui de Pascheco), livre ou écrit (le "gros volume dans lequel il y a tant d'histoires merveilleuses" dont le père d'Alphonse tirait les histoires terrifiantes).

Il s'agit là d'un élément central dans le roman de Potocki qui s'ouvre, dans la version de 1810, par le fameux "Avertissement" qui met en évidence, dès l'incipit, l'importance des motifs liés aux supports de l'écriture, à la pratique même de celle-ci, à la communication littéraire (y compris la traduction), tout cela enveloppé dans une nouvelle actualisation, fort détaillée d'ailleurs, du topos du manuscrit trouvé. Ainsi, dans sa version la plus tardive, le Manuscrit trouvé à Saragosse s'ouvre sur un "effet de réel» comportant la mention du lieu, de la date et des circonstances de la découverte du manuscrit; un «effet de réel» qui, en raison même de son caractère topique, en raison aussi du fait qu'il est absent de la version de 1804, dévoile le travail littéraire et constitue un «effet de création ${ }^{11}$.

9. Apulée, Métamorphoses 10.2-12. Aux chapitres 9-17 du premier livre des Ethiopiques d'Héliodore, on rencontre une autre belle-mère impudique dans un récit second.

Io. Apulée, Métamorphoses 3.21 ; Pétrone, Satyricon 26.

II. Pour un jeu similaire au seuil des romans grecs, je me permets de renvoyer à D. Maeder, «Au seuil des romans grecs». 
Avant de poursuivre, j'aimerais encore ajouter à ma rapide présentation du roman sa dimension spirituelle et religieuse: durant ses soixante jours passés dans la Sierra Morena, Alphonse van Worden croise des chrétiens, des musulmans et des Juifs, dont un certain Pedre de Uzeda. Cabaliste, Uzeda est destiné selon les astres à s'unir à deux épouses célestes, filles de Salomon et de la reine de Saba. Il se retrouve lui aussi dans la venta Quemada, y rencontre l'objet de ses désirs (ou plutôt les objets: elles sont sœurs jumelles) et, après une nuit d'amour divine, se retrouve à son tour le lendemain, étendu entre les deux pendus de Los Hermanos. Ainsi, quelles que puissent être leur croyance et leur religion, les personnages dans ce roman sont confrontés aux mêmes fantasmes, aux mêmes tourments et aux mêmes incertitudes: où commence la réalité?, où s'arrêtet-elle?, y a-t-il une explication rationnelle aux phénomènes surnaturels? Telles sont quelques-unes des questions que pose le Manuscrit trouvé à Saragosse.

Les rapports qui viennent d'être établis entre le Manuscrit trouvé à Saragosse et le roman antique sont évidemment partiels et partiaux: l'intertextualité est une notion fondamentalement subjective; elle dépend de l'activité interprétative de chaque lecteur, doté d'un bagage et d'une sensibilité littéraires qui lui sont propres. Il est vrai qu'en dehors d'une allusion fugitive à Psyché dans la cinquième journée ${ }^{12}$, on ne mettra le doigt sur aucune référence précise aux Métamorphoses d'Apulée, ni à un autre roman antique. Les correspondances sont vagues, d'ordre thématique essentiellement, ou relèvent, je le disais, de la technique narrative de base. Pourtant, il est avéré non seulement que Potocki savait le grec et le latin et qu'il était nourri de culture classique, mais on sait aussi qu'il avait emprunté à la bibliothèque de Wolfenbüttel, pendant l'hiver 1795, une édition des œuvres d'Apulée ${ }^{13}$. Mais l'auteur du Manuscrit trouvé à Saragosse aime à brouiller les pistes. Il tisse dans son œuvre un immense écheveau de références littéraires et de citations d'ouvrages que le chercheur est bien en peine d'identifier, parce que certaines ont été modifiées et que d'autres ont été inventées de toutes pièces. Qu'il soit

I2. Cinquième journée, MTS-1804, p. 141, MTS-1810, p. 140; le passage auquel il est fait allusion est Apulée, Métamorphoses 5.23.

I3. Voir, dans le présent volume, la contribution de Lorenz Frischknecht. 
fait mention d'œuvres de fiction ou d'ouvrages savants, Potocki semble s'amuser à travestir ses sources ${ }^{14}$.

Concernant l'Antiquité, il est cependant deux exceptions sur lesquelles j'aimerais maintenant me concentrer. Alphonse a trouvé refuge, avec l'ermite rencontré précédemment, dans le château du Juif cabaliste Uzeda. Troublé par tout ce qu'il a vu et entendu (les récits résumés ci-dessus), il s'interroge sur la nature de son aventure avec les deux femmes Maures dans la venta Quemada, cherchant en vain dans les livres de la bibliothèque des réponses à ses interrogations:

Je pris un livre dans la bibliothèque, mais je lus peu. J'étais distrait et préoccupé. Enfin on se mit à table. La conversation roula comme à l'ordinaire sur les esprits, les spectres et les vampires. Notre hôte dit que l'Antiquité en avait eu des idées confuses sous les noms d'empuses, larves et lamies, mais que les cabalistes anciens valaient bien les modernes, bien qu'ils ne fussent connus que sous le nom de philosophes qui leur était commun avec beaucoup de gens qui n'avaient aucune teinture des sciences hermétiques. L'ermite parla de Simon le Magicien, mais Uzeda soutint qu'Apollonius de Thyane devrait être regardé comme le plus grand cabaliste de ces temps-là, puisqu'il avait pris un empire extraordinaire sur tous les êtres du monde pandémoniaque. Et là-dessus, étant allé chercher un Philostrate de l'édition de Morel 1608, il jeta les yeux sur le texte grec, et sans paraître éprouver le moindre embarras à le bien comprendre, il lut en espagnol, ce que je vais raconter ${ }^{15}$.

Cet échange entre l'ermite chrétien et le Juif cabaliste débouche donc sur la lecture d'un épisode de la Vie d'Apollonius de Tyane, narrant comment le sage Apollonius avait délivré le philosophe cynique Ménipe (sic) de l'emprise d'une empuse, qui avait aveuglé ses sens en déployant autour de lui l'illusion de plaisirs terrestres. On notera le parallèle thématique entre cette anecdote et les aventures d'Alphonse, séduit non par une, mais par deux femmes, dont le jeune homme ignore si elles sont des êtres humains ou des créatures surnaturelles. On remarquera également le déploiement d'un appareil «vraisemblabilisant» avec la mention de l'édition de

I4. Sur l'intertextualité dans le manuscrit, voir J. Herman, P. Pelckmans, F. Rosset (éds), Le Manuscrit trouvé à Saragosse et ses intertextes, passim; L. Fraisse, Potocki et l'imaginaire de la création, p. 241-269.

I5. MTS-1804, p. 215 sq., MTS-1810, p. 208. 
Philostrate que l'helléniste français Frédéric Morel avait en effet publiée en 1608. Pour renforcer le sérieux de la citation-garantie, le texte de Philostrate est lu par Uzeda «dans sa version grecque originale, et traduit sur le vif en espagnol à l'intention des auditeurs " ${ }^{16}$. Signalons tout de même en passant la possibilité que Potocki ait lu cette anecdote dans l'Anatomie de la Mélancolie de Robert Burton, dont Jean Starobinski a écrit: "Une bibliothèque tient en ce livre»; il n'est pas improbable en effet que ce traité, qui fourmille d'innombrables citations d'auteurs grecs et latins notamment, ait influencé la composition du Manuscrit trouvé à Saragosse, dont on montrerait aisément qu'il est traversé par le thème de la mélancolie ${ }^{17}$.

Quoi qu'il en soit de sa source première, l'histoire d'Apollonius délivrant Ménippe de l'emprise d'une empuse se lit bel et bien dans la Vie d'Apollonius. Cette biographie romancée du philosophe pythagoricien Apollonius de Tyane est prétendument basée sur les mémoires d'un certain Damis, disciple d'Apollonius, qui aurait consigné le récit des voyages d'Apollonius sur des tablettes; ces tablettes seraient ensuite parvenues entre les mains de l'impératrice Julia qui les aurait confiées à Philostrate pour qu'il les publie. Le récit de la délivrance de Ménippe par Apollonius fait précisément partie des événements rapportés par Damis, témoin oculaire. Il y a donc, entre nos deux textes, une évidente parenté dans le déploiement des "effets de réel» autour du surnaturel. Chez Philostrate, l'épisode a pour fonction de mettre en lumière la clairvoyance du theios anèr Apollonius. Il est relaté par le narrateur principal, omniscient, qui dévoile par avance le caractère irréel de l'être ayant séduit Ménippe, et ne le remet pas en question:

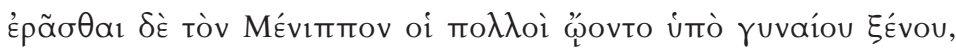

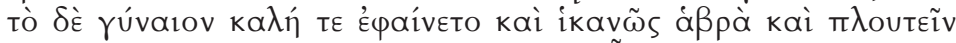

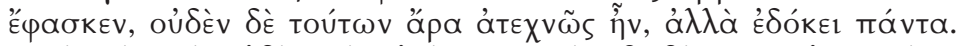

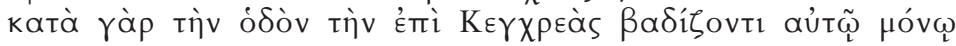

I6. F. Rosset, Le théatre du romanesque, p. 85 sq. La traduction française (due, selon MTS-1810, p. 58, au narrateur anonyme ayant découvert le Manuscrit) est basée sur la traduction française de Castillon, publiée à Berlin en 1773 avec une préface de Frédéric II de Prusse: F. Rosset, D. Triaire, MTS-1804, n. 2 p. 216, MTS-1810, p. 208.

17. Voir l'introduction de Jean Starobinski à l'édition française de 2000, qui souligne le succès immense que connut aux XVIII ${ }^{\mathrm{e}}$ et XIX $\mathrm{X}^{\mathrm{e}}$ siècles cet ouvrage paru en 1621. Le passage de Philostrate est résumé dans l'Anatomie de la mélancolie, troisième partition, section 2, membre 1, subdivision 1 (t. 2, p. 1243 sq.). 


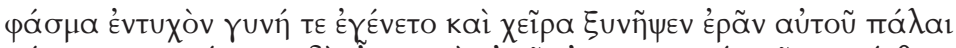

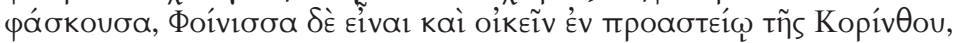

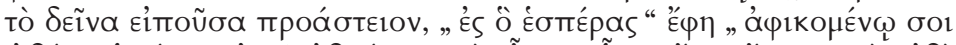

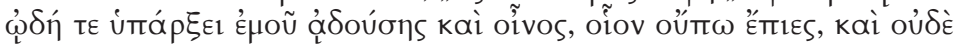

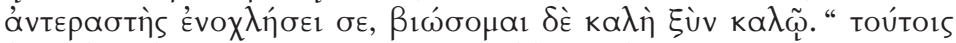

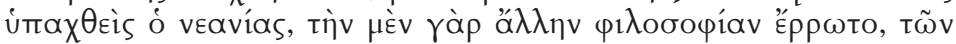

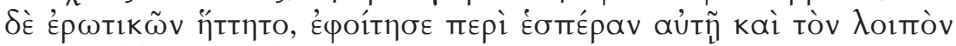

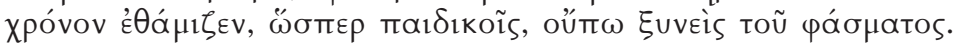

"Tout le monde croyait que ce Ménippe était aimé par une femme étrangère, qui passait pour belle, fort élégante et que l'on disait riche, alors qu'en réalité elle n'était rien de tout cela, mais n'en avait que l'apparence. Un jour qu'il se promenait, tout seul, sur la route de Cenchrae, il rencontra une apparition, qui prit la forme d'une femme: elle lui saisit la main et lui dit qu'elle l'aimait depuis longtemps, ajoutant qu'elle était phénicienne et habitait dans un faubourg de Corinthe, dont elle donna le nom. "Si tu y viens ce soir, dit-elle, tu auras ma voix qui te chantera, du vin comme tu n'en as jamais bu, et aucun rival ne viendra te déranger; et nous serons, beaux tous deux, unis pour la vie." Le jeune homme se laissa faire, car il se montrait, en tout, un excellent philosophe, mais il avait une faiblesse pour l'amour; il alla donc voir la dame, sur le soir, et, par la suite, il revint fréquemment chez elle, comme chez une maîtresse, ne comprenant pas encore que c'était seulement un fantôme.» ${ }^{18}$

Chez Potocki, l'anecdote prend une dimension fantastique, au sens todorovien du terme ${ }^{19}$. Lue par Uzeda, elle est en effet l'une des nombreuses données auxquelles Alphonse van Worden est confronté - expériences personnelles, rencontres ou lectures - qui doivent l'amener à se forger une opinion sur ce qu'il a vécu dans la venta et sur la question de l'existence ou de la non-existence d'êtres surnaturels. C'est pourquoi les interventions commentatives du narrateur omniscient dans le texte de Philostrate ont disparu: l'épisode est récrit selon la technique narrative qui domine dans le Manuscrit, celle du déploiement progressif des données narratives, sans anticipation aucune de la part du narrateur

I8. Philostrate, Vie d'Apollonius de Tyane 4.25.

19. T. Todorov, Introduction à la littérature fantastique, p. 165: «le fantastique est fondé essentiellement sur une hésitation du lecteur - un lecteur qui s'identifie au personnage principal - quant à la nature d'un événement étrange» et p. 29: «Le fantastique occupe le temps de cette incertitude; dès qu'on choisit l'une ou l'autre réponse, on quitte le fantastique pour entrer dans un genre voisin, l'étrange ou le merveilleux.» 
premier. Insérée dans la trame romanesque avec laquelle elle présente des correspondances thématiques, la mésaventure de Ménippe se plie aux stratégies qui y sont mises en œuvre. Ainsi présentée, elle se dote d'une dimension fantastique, puisque, loin de lever les hésitations d'Alphonse (et du lecteur), elle contribue à les renforcer. Ce texte de Philostrate, qui vante la clairvoyance d'un sage pythagoricien que l'on a très tôt opposé à Jésus, engage d'ailleurs un débat théologique opposant le Juif Uzeda et l'ermite. Ce dernier nie qu'Apollonius, un païen (qui plus est, né après le Christ), ait pu chasser un démon:

- Je pense, dit l'ermite, que c'était l'âme de Ménipe qu'elle voulait dévorer plutôt que son corps, et que cette empuse n'était que le démon de la concupiscence. Mais je ne conçois pas quelles étaient ces paroles qui donnaient un si grand pouvoir à Apollonius. Car enfin il n'était pas chrétien et ne pouvait user des armes terribles que l'Eglise met entre nos mains. De plus les philosophes ont pu usurper quelque puissance sur les démons avant la naissance du Christ, mais la croix qui a fait taire les oracles doit à plus forte raison avoir anéanti tout autre pouvoir des idolâtres. Et je pense qu'Apollonius, bien loin de pouvoir chasser le moindre démon, n'en aurait pas imposé au dernier des revenants, puisque ces espèces d'esprits reviennent sur la terre avec la permission divine, et cela toujours pour demander des messes, preuve qu'il n'y en avait pas au temps du paganisme ${ }^{20}$.

A cette curieuse objection quelque peu sophistique qui nie l'existence des revenants du temps des païens, Uzeda répond en brandissant l'autorité d'un second texte antique: il s'agit cette fois de la fameuse lettre de Pline le Jeune à Licinius Sura, dans laquelle Pline interroge son correspondant sur sa croyance dans les fantômes. Cette lettre permet au cabaliste d'affirmer que des revenants avaient aussi tourmenté des païens, preuve qu'il ne s'agit pas là d'un phénomène propre au christianisme:

Uzeda fut d'un avis différent: il soutint que les païens avaient été obsédés par les revenants autant que les chrétiens, bien que ce fût sans doute pour d'autres motifs; et pour le prouver, il prit un volume des Lettres de Pline où il lut ce qui suit ${ }^{21}$. 
Dans le Manuscrit, l'anecdote est introduite par un titre (absent de la traduction que De Sacy avait fait paraitre en 1722 et qui fut sans doute celle que Potocki utilisa) ${ }^{22}$. Elle relate comment le philosophe Athénagore avait loué à Athènes une maison hantée par un fantôme; loin de s'émouvoir des hurlements du spectre agitant ses chaînes, il l'avait suivi et découvert que ses ossements n'avaient pas reçu de sépulture. Dans sa lettre, Pline s'adresse à son correspondant pour lui demander s'il croit dans les fantômes:

Et mihi discendi et tibi docendi facultatem otium praebet. Igitur perquam uelim scire, esse phantasmata et habere propriam figuram numenque aliquod putes, an inania et uana ex metu nostro imaginem accipere.

«Nos loisirs nous donnent à moi la liberté d'apprendre, à vous celle d'enseigner. En conséquence, je voudrais vraiment savoir si vous croyez que les fantômes existent, ont une forme qui leur appartienne en propre et quelque puissance, ou s'ils sont sans consistance et sans réalité et ne reçoivent une apparence que de nos frayeurs. ${ }^{23}$

Pline enchaîne ensuite trois histoires qu'il a entendues (il faut souligner le caractère oral de la source d'information), qui l'amènent à croire que les fantômes existent bel et bien et que des êtres surnaturels communiquent avec les mortels pour leur transmettre un message. La première histoire concerne Curtius Rufus, auquel une femme surnaturelle se présentant comme étant l'Afrique avait prédit l'avenir glorieux et la fin malheureuse; la seconde est l'anecdote de la maison hantée reprise par Potocki; la dernière, une aventure arrivée à un affranchi de Pline que le grand homme (quelque peu égocentrique) interprète comme un signe surnaturel lui annonçant qu'il avait échappé à une accusation sous Domitien ${ }^{24}$. Trois récits merveilleux, donc, qui nécessitent d'être authentifiés, ce que Pline a soin de faire: le premier est introduit par l'expression: Ego ut esse

22. F. Rosset, D. Triaire, $M T S-1804$, n. 1 p. 220 , MTS-1810, n. 1 p. 212 , qui mentionnent une édition de 1752. L'anecdote est évoquée par Robert Burton dans l'Anatomie de la mélancolie, première partition, section 2, membre 1, subdivision 1 (t. 1 , p. 319).

23. Pline le Jeune, Correspondance 7.27.1.

24. On notera en passant que Pline le Jeune s'oppose ici à l'incrédulité exprimée par son oncle naturaliste au début du livre 30 de l'Histoire naturelle à l'encontre des pratiques magiques et des prédictions d'avenir. 
credam in primis eo ducor, quod audio accidisse Curtio Rufo. Le second par la phrase: Iam illud nonne et magis terribile et non minus mirum est, quod exponam ut accepi? Enfin, Pline passe à l'événement qui le concerne en remarquant: Et haec quidem adfirmantibus credo; illud adfirmare aliis possum.

De ces trois anecdotes, Potocki ne conserve que la deuxième. Gommant la forme épistolaire, il ne retient rien non plus des questionnements et des incertitudes de Pline. Le récit de la maison hantée est présenté comme un fait avéré, une preuve de l'existence des fantômes du temps des païens, fournie par un texte antique. Pour en renforcer le caractère vénérable, Potocki transforme ce qui, chez l'auteur romain, était présenté comme une relation orale en une source de garantie écrite. Comme le premier texte lu par Uzeda, la respectabilité de cet exemplum tient également au fait qu'il concerne une aventure survenue à un sage.

Arrêtons-nous un instant sur le nom de ce personnage courageux et clairvoyant. Selon Pline, il s'agit d'Athénodore, probablement le philosophe stoïcien originaire de Tarse, ami d'Auguste ${ }^{25}$. Selon Potocki, il s'agit d'Athénagore, peut-être le philosophe platonicien du II ${ }^{\mathrm{e}}$ siècle de notre ère, dont la conversion au christianisme est évoquée par Philippe de Sidè ( $\mathrm{V}^{\mathrm{e}}$ siècle) et qui «fut amené à la foi des chrétiens par la lecture de leurs livres qu'il avait étudiés pour les combattre» ${ }^{26}$. La probabilité de cette identification est renforcée par le fait qu'Athénagore a rédigé un traité De resurrectione, ce qui démontre son intérêt pour l'après-vie ou l'après-mort. Quoi qu'il en soit, cette confusion autour du nom du héros de cette histoire n'est pas sans précédent: la même anecdote se lit dans les Amis du mensonge de Lucien ( $\$ 30$ et suivants), un traité qu'on peut rapprocher du Manuscrit trouvé à Saragosse notamment en ce qui concerne l'enchaînement de récits extraordinaires narrés à la première personne par un groupe de sages philosophes; on y trouve aussi le thème de la crédulité versus l'incrédulité. Or dans ce texte, c'est le philosophe pythagoricien Arignotus qui délivre une maison du fantôme qui la hantait, à Corinthe. Lucien nous livre le (prétendu) récit d'Arignotus, témoin

25. A. N. Sherwin-White, The letters of Pliny, p. 436 ad loc.

26. F. Rosset, Le théatre du romanesque, p. 86, qui cite l'article "Athénagore» de la Grande Encyclopédie, Paris 1885-1902; sur Athénagore, voir B. Pouderon, Athénagore d'Athènes, philosophe chrétien et Athénagore, Supplique au sujet des chrétiens et Sur la résurrection des morts. 
oculaire, racontant lui-même, à la première personne, sa confrontation avec le fantôme. Pour en revenir au Manuscrit, François Rosset remarque fort justement que la confusion fortuite ou volontaire entre le nom donné par Pline et celui donné par Potocki «met en lumière le substitut Athénagore», un homme converti au christianisme par la lecture des textes chrétiens ${ }^{27}$. On a ainsi dans le Manuscrit une sorte d'inversion intriquée, raffinée et amusante: un Juif cabaliste avance l'autorité d'un texte païen (la lettre de Pline) relatant une aventure survenue à un philosophe grec converti au christianisme par la lecture des textes chrétiens (Athénagore) pour convaincre ses interlocuteurs (l'ermite, Alphonse) qu'il existait des fantômes du temps des païens.

Il reste à se demander si ces deux histoires de fantômes surgies de l'Antiquité, avec leur vénérable préséance, aident Alphonse à y voir plus clair dans sa mésaventure. Dans la version de 1810, la vérité sur son expérience lui sera dévoilée à la toute fin du roman, lors de la soixantième journée, par l'ermite qui, de façon pour le moins inattendue, se révèle être le grand scheik des Gomelez, un mahométan... L'habit ne fait décidément pas l'ermite. Quant au Juif Uzeda, il apparaît être Mamoun, son vassal. Alphonse apprend ainsi qu'il a été le jouet d'une cabale destinée sinon à le convertir, du moins à mettre à l'épreuve sa loyauté à coup de narcotiques, de mises en scène théâtrales et de récits affabulateurs (récit de Pascheco et d'Uzeda). Ces révélations, aussi précipitées qu'alambiquées, ont pour effet d'évacuer le fantastique de la dernière version du Manuscrit au profit d'un rationnel guère moins alambiqué ${ }^{28}$. Rétrospectivement, elles font du débat opposant Uzeda et «l'ermite» sur

27. F. Rosset, Le théâtre du romanesque, p. 86; p. 87: «Le nom d'Athénagore dans notre Manuscrit soi-disant trouvé à Saragosse, qu'il soit le résultat d'une erreur ou d'une manœuvre consciente, vient rappeler - peut-être bien malgré lui - que la littérature est un corps organique dont toutes les parties, réciproquement, dépendent les unes des autres, s'engendrent les unes les autres et se répondent au-delà des frontières temporelles, spatiales, idéologiques ou génériques. Lisant dans les livres, les personnages du Manuscrit remontent à la source qui leur a donné vie, rejoignent et invoquent en même temps les personnages et les livres antérieurs, suggèrent les personnages et les livres à venir.»

28. A vrai dire, plusieurs indices suggèrent que le rationnel trouvera sa place dans les aventures d'Alphonse: p. ex. MTS-1804, p. 197 sq., où Alphonse se livre à des réflexions qui se révéleront bien proches de la vérité dévoilée dans la version de 1810 ; cf. aussi MTS-1810, p. 191 et 596. Dans les deux versions, l'histoire fantastique du chevalier de Tolède trouvera une explication rationnelle lorsqu'elle recoupera celle de Lope Soarez. 
la question des fantômes une farce et jettent le discrédit sur les textes antiques évoqués. Les livres seraient-ils donc une source de connaissance moins fiable que les récits oraux? C'est apparemment le cas, pour autant qu'Alphonse (et le lecteur) apprenne à distinguer les récits mensongers des révélations véridiques. Dans la version de 1804, inachevée, l'autorité des Anciens n'est en revanche pas remise en question. Il revient à Alphonse (et au lecteur) de se forger une opinion en croisant les différentes sources d'information, écrites, orales, qui s'entremêlent. S'il peut soupçonner une explication rationnelle, du moins les références aux textes de Philostrate et de Pline n'interdisent-elles pas le fantastique.

Ainsi, extraits de leur rayonnage, les ouvrages des Anciens, avec leur autorité millénaire, participent au même titre que les récits oraux enchâssés à la construction d'un univers dans lequel Alphonse doit apprendre à distinguer le vrai du faux, le réel de l'imaginaire - ou du construit. Les références à l'Antiquité dont il a été question dans cette étude font partie d'une immense bibliothèque de citations et d'allusions qui, dans la version de 1804 comme dans celle de 1810, ont pour effet de déclarer la texture livresque du Manuscrit trouvé à Saragosse.

Danielle van Mal-Maeder

Université de Lausanne 


\section{BIBLIOGRAPHIE}

\section{Textes}

Burton, Robert, Anatomie de la mélancolie, traduction de Bernard Hoepffner, Catherine Goffaux, préface de Jean Starobinski, postface de Jackie Pigeaud, Paris, José Corti, 2000.

MTS-1804 et MTS-1810 = Pотоскі, Jean, Manuscrit trouvé à Saragosse (version de 1804 et version de 1810), éd. par François Rosset, Dominique Triaire, Paris, Flammarion, 2008 (GF 1342-1343).

Philostrate, La vie d'Apollonius de Tyane, in Romans grecs et latins, traduction de Pierre Grimal, Paris, Gallimard, 1958.

Pline le Jeune, Correspondance. Livres VII-IX, texte établi et traduit par Anne-Marie Guillemin, Paris, Les Belles Lettres, 2003 (1928¹).

\section{Travaux}

Barthes, Roland, S/Z, Paris, Le Seuil, 1970.

Fraisse, Luc, Potocki et l'imaginaire de la création, Paris, PUPS, 2006.

Herman, Jan, Pelckmans, Paul, Rosset, François (éds), Le Manuscrit trouvé à Saragosse et ses intertextes. Actes du colloque international, Leuven-Anvers, 30 mars - $1^{\text {er }}$ avril 2000, Louvain, Peeters, 2001.

Maeder, Danielle, "Au seuil des romans grecs. Effets de réel et effet de création", in Groningen Colloquia on the Novel 4, ed. by Heinz Hofmann, Groningen, E. Forsten, 1991, p. 1-33.

Mal-Maeder, Danielle van, Apuleius Madaurensis Metamorphoses, livre II. Texte, introduction et commentaire, Groningen, E. Forsten, 2001.

Pouderon, Bernard, Athénagore d'Athènes, philosophe chrétien, Paris, Beauchesne, 1989. 
-, Athénagore, Supplique au sujet des chrétiens et Sur la résurrection des morts. Introduction, texte et traduction, Paris, Les Ed. du Cerf, 1992.

Rosset, François, Le théâtre du romanesque. Manuscrit trouvé à Saragosse entre construction et maçonnerie, Lausanne, L'Age d'homme, 1991.

Rosset, François, Triaire, Dominique, Jean Potocki. Biographie, Paris, Flammarion, 2004.

SHERWin-White, Adrian Nicholas, The letters of Pliny: a historical and social commentary, Oxford, Clarendon Press, 1966.

Stramaglia, Antonio, Res inauditae, incredulae. Storie di fantasmi nel mondo greco-latino, Bari, Levante, 1999.

Todorov, Tzvetan, Introduction à la littérature fantastique, Paris, Seuil, 1970.

Winkler, John, Auctor \& Actor. A Narratological Reading of Apuleius' The Golden Ass, Berkeley/Los Angeles/London, Univ. of California Press, 1985. 
